\title{
Comparison of Different Non-invasive $\mu$-Raman Set-ups for Studying Pigments in Art Works
}

\author{
Cristina Aibéo*1, Stefan Röhrs², Giorgia Marucci ${ }^{1,2}$, Ina Reiche ${ }^{1}$ \\ 1. Rathgen Research Laboratory, National Museums in Berlin, Prussian Cultural Heritage Foundation, Berlin, Germany \\ 2. Dept. of Applied Sciences, North Umbria University, Newcastle City Campus, United Kingdom
}

\begin{tabular}{|c|c|}
\hline Article Info & ABSTRACT \\
\hline $\begin{array}{c}\text { Received: 2017/03/20; } \\
\text { Accepted: 2017/05/21 } \\
\text { Published Online 2017/10/29 }\end{array}$ & $\begin{array}{l}\text { Micro-Raman spectroscopy is widely used for the identification of pigments on ar } \\
\text { works. Due to the priceless value of artistic objects, non-invasive measurements art } \\
\text { strongly preferred over sampling strategies. However, the non-invasive spectra } \\
\text { response is often lower if compared to measurements carried out on a sample. In this } \\
\text { study, spectral intensity of measurements performed with various non-invasive Ramar } \\
\text { set-ups was compared with spectral intensity from a traditional micro-Raman set-up, } \\
\text { The comparison was done on pure pigments reference swatches first, to test th } \\
\text { methodology. Then a painting was investigated, as case study on a real artefact, directly } \\
\text { on its surface and on taken samples under the microscope. For the non-invasivt } \\
\text { measurements, four horizontal beam set-ups and an external measuring head connectec } \\
\text { to the spectrometer by fibre optics were used. Two lasers were used: } 785 \text { and } 532 \text { nm } \\
\text { The results were determined as counts (Raman signal intensity) per second o: } \\
\text { measurement and per Watt of laser intensity (Cnts/Ws). A method to compart } \\
\text { performances among the different experimental conditions is proposed in this work } \\
\text { The horizontal beam configurations showed spectral responses in signal intensity very } \\
\text { similar to those of the traditional microscope but the ones from the fibre optics wert } \\
\text { only at about } 10 \% \text { of that of the microscope. These results show the potential o: } \\
\text { horizontal set-ups for the analyses of artworks, as their performance is equivalent tc } \\
\text { the traditional microscope configuration. However, the more challenging positioninধ } \\
\text { for the horizontal beam set-ups can hamper the acquisition of a good spectrum, sinct } \\
\text { either the Raman spectrometer or the artwork has to be moved with } \mu \text { m scale precisior } \\
\text { to position the laser spot on the area of interest. This often requires improvements } \\
\text { especially regarding the mechanical stability of the analytical set-up as well as th } \\
\text { artwork. }\end{array}$ \\
\hline
\end{tabular}

Keywords: Raman Spectroscopy, Pigments, Non-Invasive, in situ Analyses, Signa Intensity

Corresponding Information: Rathgen Research Laboratory, National Museums in Berlin, Prussian Cultural Heritage Foundation, Berlin, Germany. Tel. +49 (0)30266427142, Email: c.aibeo@smb.spk-berlin.de

Copyright ( ) 2017, Ir Cons Sci J. This is an open-access article distributed under the terms of the Creative Commons Attribution-noncommercial 4.0 International License which permits copy and redistribute the material just in noncommercial usages, provided the original work is properly cited.

\section{Introduction}

Raman-spectroscopy is one of the most imported methods for the identification of pigments in paintings (Clark, 2002; Smith \& Clark, 2004; Vandenabeele et al., 2007) or other works of art (Deneckere, Schudel, Van Bos, Wouters, \& Bergmans, 2010). Indeed, pigments are high Raman active compounds that makes micro-Raman spectroscopy an ideal tool to identify them, both inorganic and organic ones, on ancient as well as on contemporary objects. The analysis of pigments is used to study art works for questions of technical art history or forensic sciences.
No matter the reason of the study of an artwork, a nonsampling approach and, if possible, in situ measurements are strongly preferred over sampling the artwork. The second important advantage of completely non-invasive measurements is that all accessible areas of the painting can be analysed, whereas samples can only be collected from limited, and thus less representative areas (Deneckere et al., 2010; Vandenabeele et al., 2005, 2007).

Depending on the analytical circumstances, the most suitable working set-up needs to be chosen: the traditional 
Raman microscope, the horizontal beam with extension tubes of different length or the measuring head with fibre optic. If the object is small enough, e.g. watercolours or miniature paintings (Röhrs \& Stehr, 2014), non-invasive measurements can be carried out directly under the microscope with its traditional set-up. In this configuration, the object can also be easily moved ( $\mathrm{x}-$, $\mathrm{y}-$ and $\mathrm{z}$-wise) with a motorized microscope stage and the focusing is fast and straight forward.

However, if the objects dimensions do not allow such a measurement, the object needs to be placed in front of the instrument. Typically, the non-invasive set-up of a laboratory instrument directs the laser beam onto the sample either by deflecting the laser by a mirror or by guiding it via fibre optics. Using the deflecting mirror, an extension tube of adapted length which holds the objective is required to focus the laser on the surface of the object. If an object cannot be placed in front of to the instrument, e.g. a mural painting, a measuring head, which is connected to the spectrometer via fibre optics can be used alternatively. This fibre can guide the light of the laser though a several meters long cable to the measuring head, where the focusing optics are installed. In this case, the measuring head must be positioned precisely to focus on the chosen spot.

Many publications compare between bench and portable Raman spectrometers, but the performances of non-invasive set-ups of a laboratory instrument were seldom discussed in publications. Portable instruments often show some disadvantages such as: 1) they provide a narrow spectral window (Bersani et al.,2006. PerezAlonzo et al., 2004, Reiche et al.,2004), sometimes with a cut-off filter at $200 \mathrm{~cm}-1 ; 2$ ) often there are fewer lasers available (Aceto et al., 2012; Bersani et al., 2006), frequently only one, or 3) they provide lower laser power (Aceto et al., 2012; Boschetti, Corradi, \& Baraldi, 2008; Trentelman \& Turner, 2009). When comparing the Raman response, portable set-ups also show in general a lower signal to noise ratio (Ziemann, 2006).

Nevertheless, the aim of this study is to obtain a better understanding of the loss of signal by using our noninvasive micro-Raman laboratory device in comparison to the traditional microscope set-up. This will help us to choose the most appropriate spectrometer configuration for a given analytical problem. For the performance tests, measurements were carried out on pure pigment reference samples and, as a case study, non-invasively on a painting and on samples from the same painting.

\section{Material and Methods}

\subsection{Pure pigments (reference samples)}

The reference samples consisted of pellets of pure pigments: cinnabar, red ochre, ultramarine blue, yellow chrome and copper phthlalocyanine blue. The choice of the pigments relied on their homogeneity and presence in the case study painting.

Pure pigments were prepared as pellets. The small size of the pellets (13 $\mathrm{mm}$ diameter) made the spectra acquisition straightforward. The samples were mounted on a sample holder, to easily move from one pellet to the next.

Six pigments were initially chosen to estimate the spectroscopic performance of the different set-ups: ultramarine blue $\left(\mathrm{Na}_{8-10} \mathrm{Al}_{6} \mathrm{Si}_{6} \mathrm{O}_{24} \mathrm{~S}_{2-4}\right)$, chrome yellow $\left(\mathrm{PbCrO}_{4}\right)$, cinnabar $(\mathrm{HgS})$ and minium $\left(\mathrm{Pb}_{3} \mathrm{O}_{4}\right)$ from Kremer Pigments, anatase $\left(\mathrm{TiO}_{2}\right)$ from Hombitan and phthalocyanine blue $\left(\mathrm{C}_{32} \mathrm{H}_{16} \mathrm{CuN}_{8}\right.$ - PB15:1 - copper phthalocyanine) from BASF (Heliogen Blau L 6930).

These were prepared as pellets using a pellet-making die. Since a pellet of pure pigment would be too brittle, the pellet has a second layer of cellulose and Plexigum in extremely low concentration is used as pigment binder. No trace of cellulose or Plexigum was seen in the Raman spectra. For each pigment's preparation the following method was followed:

1. $100 \mathrm{mg}$ of cellulose (Somar Mix) were weighted on the pellet making die and pressed for ca. $20 \mathrm{~s}$ with $3 \mathrm{t}$.

2. $50 \mathrm{mg}$ of pure pigment were well mixed in an agate mortar with $150 \mu \mathrm{L}$ of a $2 \%$ (w/w) solution of Plexigum (Polymer in powder form based on iso-butyl methacrylate), let to dry and mixed again.

3. This mixture was added to the pellet-making die (on top of the cellulose layer) and pressed at $8 \mathrm{t}$ for ca. $20 \mathrm{~s}$.

Homogeneity of the pure pigment's references was tested by Environmental scanning electron microscopy (ESEM) with an energy-dispersive X-ray analyser (EDX).

\subsection{Case study painting}

The case study concerns a falsified painting. The painting "Cubist still life" (1913) had been wrongly believed to be by Fernand Léger (1881-1955) and is painted with oil on canvas (H $55.4 \mathrm{~cm} \mathrm{x} \mathrm{B} 38.0 \mathrm{~cm}$ ). Samples of the painting had already available in the Rathgen Research Laboratory from analysis carried out in the past.

The presence of synthetic pigments evidenced in samples from the painting allowed excluding the year 1913 as the proposed production date. Fig 1 shows the painting with sampling positions from the previous measurement campaign and the area analysed by non-invasive analyses (this study). 


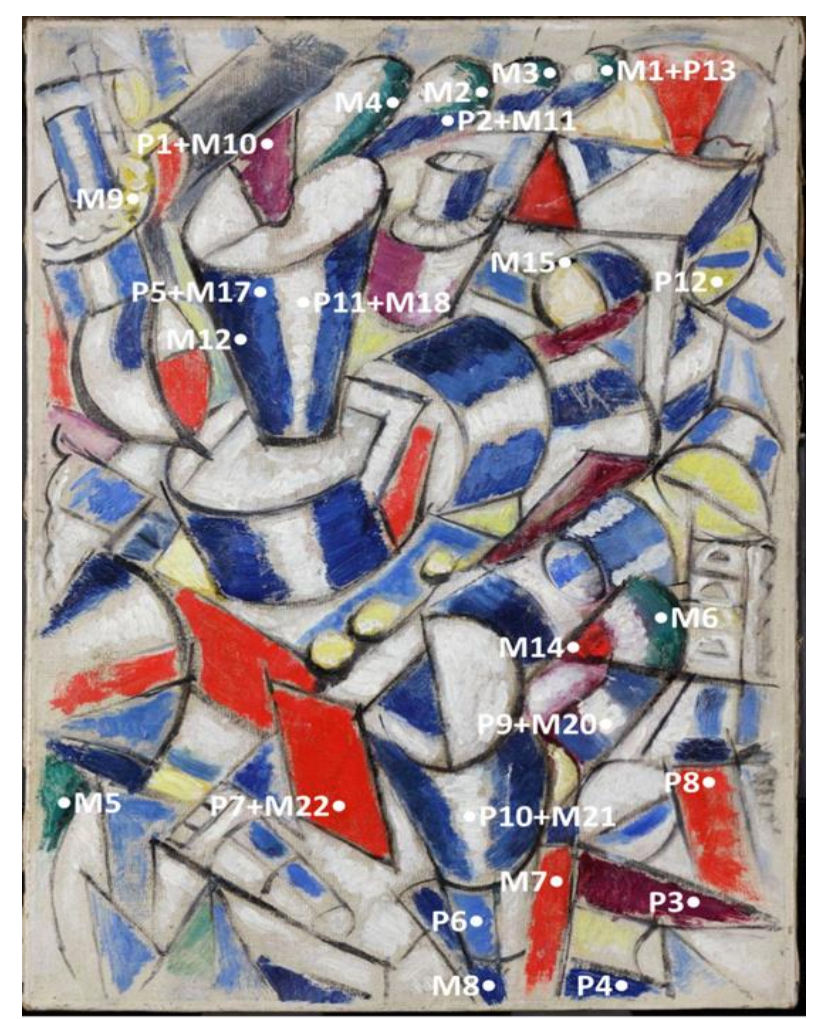

Fig 1. Cubistic still life, oil on canvas, formerly attributed to Fernand Léger, $(55.4 \mathrm{~cm}$ x $38.0 \mathrm{~cm})$. P no.: sampled spots; M no.: non-invasively measured spots.

\section{Experimental}

\subsection{Environmental scanning electron microscopy (ESEM) with an energy-dispersive $\mathrm{X}$-ray analyser (EDX)}

A Quanta 200 environmental scanning electron microscope from Fei equipped with an energy-dispersive X-ray analyser (Flash X 4010 Bruker) was used to map element distributions. Measurements were carried out in an atmosphere of 0.75 torr of water vapour to prevent electrical charging of the sample surface.

To test the homogeneity of the pellets of pure pigments, the distribution of the main elements was measured by an EDX chemical mapping of the ROI (Region of Interest) of characteristic $\mathrm{x}$-ray lines.

\subsection{Raman spectroscopy}

A Horiba XploRa Micro-Raman system, equipped with a $532 \mathrm{~nm}$ and a $785 \mathrm{~nm}$ laser, was used. The maximum power of each source is $25 \mathrm{~mW}$ and $90 \mathrm{~mW}$ respectively. The excitation wavelength influences, for example, the spectral response of a certain pigment and fluorescence interference, hence, different lasers were used to increase the signal-to-noise ratio. The laser power is controlled using different filters. The reference samples were measured with filters with transmissions of $1 \%$ or $10 \%$ and the painting with $1 \%, 10 \%$ and $25 \%$. The spectral resolution is managed through different gratings. The grating used was 1200 lines $/ \mathrm{mm}$. The spatial resolution is ca. $2 \mu \mathrm{m}$ in the plane of the sample surface.

A 50x objective with long focal length was used (Olympus LMPlanFLN50x/0.50, $\infty / 0 / \mathrm{FN} 26.5$ ). For the fibre optics set-up, a 20x objective (Olympus MPlanFNL $20 \mathrm{x} / 0.45, \infty / 0 / \mathrm{FN} 26.5$ ) was used with the microscope to focus laser into the glass fibre and the 50x with the videohead.

Time of acquisition varied between 0.1 and $20 \mathrm{~s}$ and cycles (accumulations) between 1 and 20. Wavelength scale calibration was carried out using a $\mathrm{Si}$ standard $\left(520.5 \mathrm{~cm}^{-1}\right)$. Data acquisition and spectra elaboration was performed with NGSLabSpec software. "SuperHead" fibre probes from Horiba for both lasers were also used. With these set-ups, the laser visible light is connected via optic fibre to the remote optical head and a second fibre connects the head to the spectrometer. All measurements were performed in darkness.

To obtain the pigments intensity of the Raman signal the baseline was subtracted from each spectrum and the amplitude of the strongest band in counts was compared. The results were determined as counts (Raman signal intensity) per second of measurement and per Watt of laser intensity (Cnts/Ws). Parameters, such as intensity, objective, grating, time of acquisition and accumulations were kept constant whenever possible. Several sets of measurements were made for the reference samples. Each of them consisted in: 1) measuring the standard crystalline silicon sample to verify the intensity of its characteristic Si$\mathrm{O}$ stretching band at ca. $520 \mathrm{~cm}^{-1}$;2) measuring all the samples with all the set-ups available and 3) measure again the silicon standard to ascertain that the intensities of the lasers did not change during the session. Only sets of measurements (three in total) without significant change in the intensity of the silicon band $(<5 \%)$ were considered. All the values presented here are an average of the three measurements.

The traditional microscope setup (configuration 1 in Fig 2), was compared with setups for analyses directly on the object (configurations 2a-d in Fig 2), where the laser path is deviated by $90^{\circ}$ (using a so-called macro accessory which contains a $45^{\circ}$ sloped mirror) and with the fibre optics setup, which allows the examination of an object few metres away from the Raman instrument (configuration 3 in Fig 2).

Table 1 summarises the characteristics of the setups that are schematised in Fig 2 
Table 1: Summary of the different set-ups

\begin{tabular}{|c|c|c|c|c|}
\hline Setup & \multicolumn{3}{|r|}{ Set-up characteristics } & Suitable for: \\
\hline 1 & \multicolumn{3}{|c|}{ traditional microscope configuration } & $\begin{array}{l}\text { collected samples or small, } \\
\text { flat and thin objects }\end{array}$ \\
\hline \multirow{4}{*}{$2 \mathrm{a}-\mathrm{d}$} & \multirow{4}{*}{$\begin{array}{l}90^{\circ} \text { deviated } \\
\text { laser path by } \\
\text { a } 45^{\circ} \text { sloped } \\
\text { mirror }\end{array}$} & a & macro accessory & flat surfaces of small objects \\
\hline & & $\mathrm{b}$ & macro accessory + self-made extension tube & flat surfaces \\
\hline & & $\mathrm{c}$ & macro accessory + commercial extension tube & \multirow{3}{*}{$\begin{array}{l}\text { any desired surface spot, as } \\
\text { long as it is possible to focus } \\
\text { the laser on it }\end{array}$} \\
\hline & & $\mathrm{d}$ & commercial stable extension tube & \\
\hline 3 & & & fibre optics' & \\
\hline
\end{tabular}

As evidenced in Fig 2, due to the microscope design, configuration $2 \mathrm{a}$ does not allow the measurements of spots more than ca. $15 \mathrm{~cm}$ away from the border of the painting, because of the design of the Horiba system. Only for small paintings (ca. $30 \mathrm{~cm}$ ) without frame, it is possible to reach all positions. Extension tubes allow the analyses of broader and bulkier objects.

Therefore, an extension tube of $3 \mathrm{~cm}$, placed between the $45^{\circ}$ mirror piece and the objective, was self-made (configuration 2b in Fig 2). With this extra accessory, it is possible to analyse canvas (or any other flat object) of any size. There are two types of extension tubes (ca. $20 \mathrm{~cm}$ ) commercially available. These were also tested and are represented in Fig 2. A simple tube, that can be screwed to the macro accessory (Fig 2c) and an extension, which includes the $45^{\circ}$ inclination mirror and is attached directly to the microscope (Fig 2d). The later solution seems to be mechanically more stable as is it fixed in the mounting for the revolving nosepiece.

For the fibre optics' configuration set-up, as seen in Fig 2 configuration 3 . This configuration is the most versatile as it allows the analyses of objects with almost any shape.

The painting was analysed only with the $785 \mathrm{~nm}$ laser since the Raman spectra acquired from the collected samples in the preceding analysis were acquired only with this laser and comparisons are only made using the same laser. Additionally, not all sampling spots (P) were accessible with all set-ups (e.g. configurations $2 \mathrm{a}$ and $2 \mathrm{~b}$ in Fig 2).

Further restrictions have been implied by the absence of the $785 \mathrm{~nm}$ measuring head and the simple commercial extension tube (configuration $2 \mathrm{c}$ in Fig 2) due to organisations issues for the measurements of the reference sample.

Samples from the "Cubist still life" were collected and analysed with the experimental configuration 1 . The sampling spots are marked as "P" in Fig 1. Subsequently, the painting was analysed with the non-invasive set-ups, as seen in Fig 3 using the $785 \mathrm{~nm}$ laser, since the $532 \mathrm{~nm}$ laser caused too high fluorescence signals. The analysed spots are marked ' $M$ ' in Fig 1 . With configuration 2a) it was not possible to reach all spots in the painting as the painting was larger than $30 \mathrm{~cm}$.
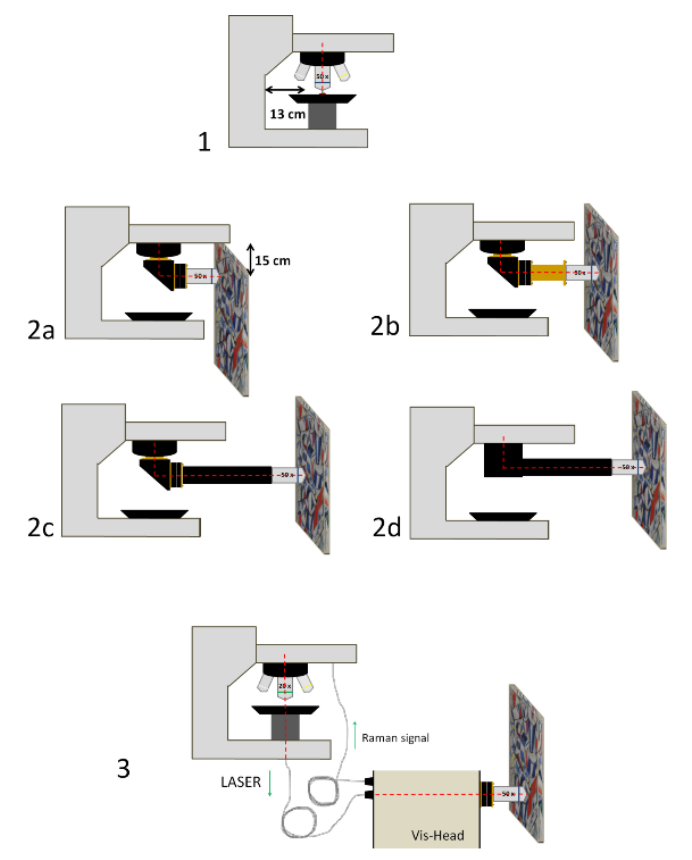

Fig 2. Schematic views of configuration 1: traditional microscope setup with the 50x objective; 2 : with the a) macro accessory ( $45^{\circ}$ mirror) and b) the short extension tube; c) the simpler commercial tube and d) the more stable one and 3: measuring head connected via fibre optics.

\subsection{Raman setup performance evaluation procedure}

It has been proposed to use the limit of detection (LOD) as a parameter to compare two different experimental approaches (Vandenabeele, 2013; Vandenabeele \& Moens, 2012). In the following, a procedure to calculate the LOD is proposed with the intention to obtain a value to compare experimental setups. The authors are aware of the fact that the calculated values may not represent the realistic detection limit given in concentration. 
Generally, the smallest signal that can be identified with certainty as a peak in a spectrum should be three times larger than the fluctuation of the total background (shot noise) at the peak position. In counting statistics, i.e. spectra, the fluctuations of the background are equal to the square root of the background. The spectral background is generated by the total shot noise, which contains contributions of scattered light and background fluorescence radiation from the matrix in the analytical volume and noise from various sources. The background subtraction (eq.2) was used to determine the intensity of the background, $I_{B}$, by the difference of the intensity of the peak before the background subtraction, $I_{p}$, and the intensity of the Raman signal, $I_{R}$, after the background subtraction.

$$
\begin{aligned}
& I_{P}=I_{B}+I_{R} \\
& I_{B}=I_{P}-I_{R}
\end{aligned}
$$

The minimum intensity of a detectable peak sitting on a background of $\mathrm{I}_{\mathrm{B}}$ is therefore given as

$$
L O D_{I}=3 \sqrt{I_{B}} \quad \text { (eq. } 3 \text { ) }
$$

The $\mathrm{LOD}_{\mathrm{C}}$ in concentration is calculated by equation 4 , where $b$ denotes the response of the spectrometer on a specific peak of a compound in Cnts/wt\%. In quantitative analytical this value is obtained from the calibration curve where $\mathrm{b}$ is the slope of the curve.

$$
\left.L O D_{C}=\frac{3 \sqrt{I_{B}}}{b} \quad \text { (eq. } 4\right)
$$

As an estimation of $b$ the $I_{R}$ values of the measurements of the pure pigments was used assuming a straight calibration curve from the concentration at zero to the measured value for a concentration of $100 \%$ of the pigment. The $\mathrm{b}$ value for the pure pigment can be calculated as given in the following equation, with $x$ indexing the instrumental set up used:

$$
b_{\text {pure }}^{x}=\frac{I_{R}^{x}}{100 w t \%}
$$

For the measured spectra of anatase the values for $\mathrm{LOD}_{\mathrm{I}}, \mathrm{LOD}_{\mathrm{C}}$ and $b_{\text {pure }}^{x}$ are given in Table 2 . The given values were derived from spectra where the measurement time and accumulations where not always kept constant. The parameters times and accumulations have been adapted for each set up in order to obtain the best possible spectra from the analysed volume, in the way as an operator would work analytical measurements on an art work.

The limit of detection (LOD) was calculated for anatase assuming $I_{P}$ is the signal (counts) of the strongest peak $\left(143 \mathrm{~cm}^{-1}\right)$ before baseline subtraction and $I_{R}$ after subtraction. With values $\mathrm{I}_{\mathrm{B}}$ and $\mathrm{b}$ the LOD was calculated according to the following equation.

$$
\left.L O D_{C}=\frac{3 \sqrt{I_{B}}}{b_{\text {pure }}} \quad \text { (eq. } 6\right)
$$

The $\mathrm{LOD}_{\mathrm{C}}$ values can be used for a direct comparison of the set-ups whether the $\mathrm{LOD}_{\mathrm{I}}$ values give an indication of the peak high necessary for a detectable signal. It is important to be aware of this difference as a configuration with a poor sensibility (as configuration 3 in our study) can have a low LOD $_{\text {I }}$ but a high LOD $_{C}$. To avoid misunderstandings we like to emphasize that we do not believe that the $\mathrm{LOD}_{\mathrm{C}}$ values are obtained in a way, which would allow using them as a reliable value for the actual limit of detection but are for reference only. The $\mathrm{LOD}_{\mathrm{C}}$ values are not believed to give a realistic limit of detection of a setup but are used as index to compare the performance of the set-ups.

\section{4. . Results and Discussion}

Measuring reference pure pigments prepared as homogeneous and flat pellets does not represent a real case study but allowed in a simple way to characterize the sensitivity of in-situ set-ups, which was the aim of this study.

\subsection{Results on reference samples by Environmental scanning electron microscopy (ESEM) with an energy-dispersive $\mathrm{X}$-ray analyser (EDX)}

Before the Raman measurements, the pellets of anatase, chrome yellow, cinnabar, minium, phthlalocyanine blue and ultramarine were analysed with the ESEM-EDX to ascertain that their composition was homogeneous and not contaminated.

As it can be seen from the ESEM chemical maps in, elements in the ultramarine pellet are heterogeneously distributed. For example, Ca-rich grains (probably calcite) of ca. $10 \mu \mathrm{m}$ diameter are present. These are larger than the Raman spot size (about $2 \mu \mathrm{m}$ ) and their heterogeneous distribution could lead to very different bands or bands' intensities in the Raman spectra depending on the actual spot analysed. Therefore, this sample was discarded.

\subsection{Raman spectroscopy results of reference samples}

The spectra obtained for phthalo-cyanine are shown as example in Fig 4 (Bell, Clark, \& Gibbs, 1997; Bouchard \& Smith, 2003; Burgio \& Clark, 2001; Scherrer, Zumbuehl, Delavy, Fritsch, \& Kuehnen, 2009). 


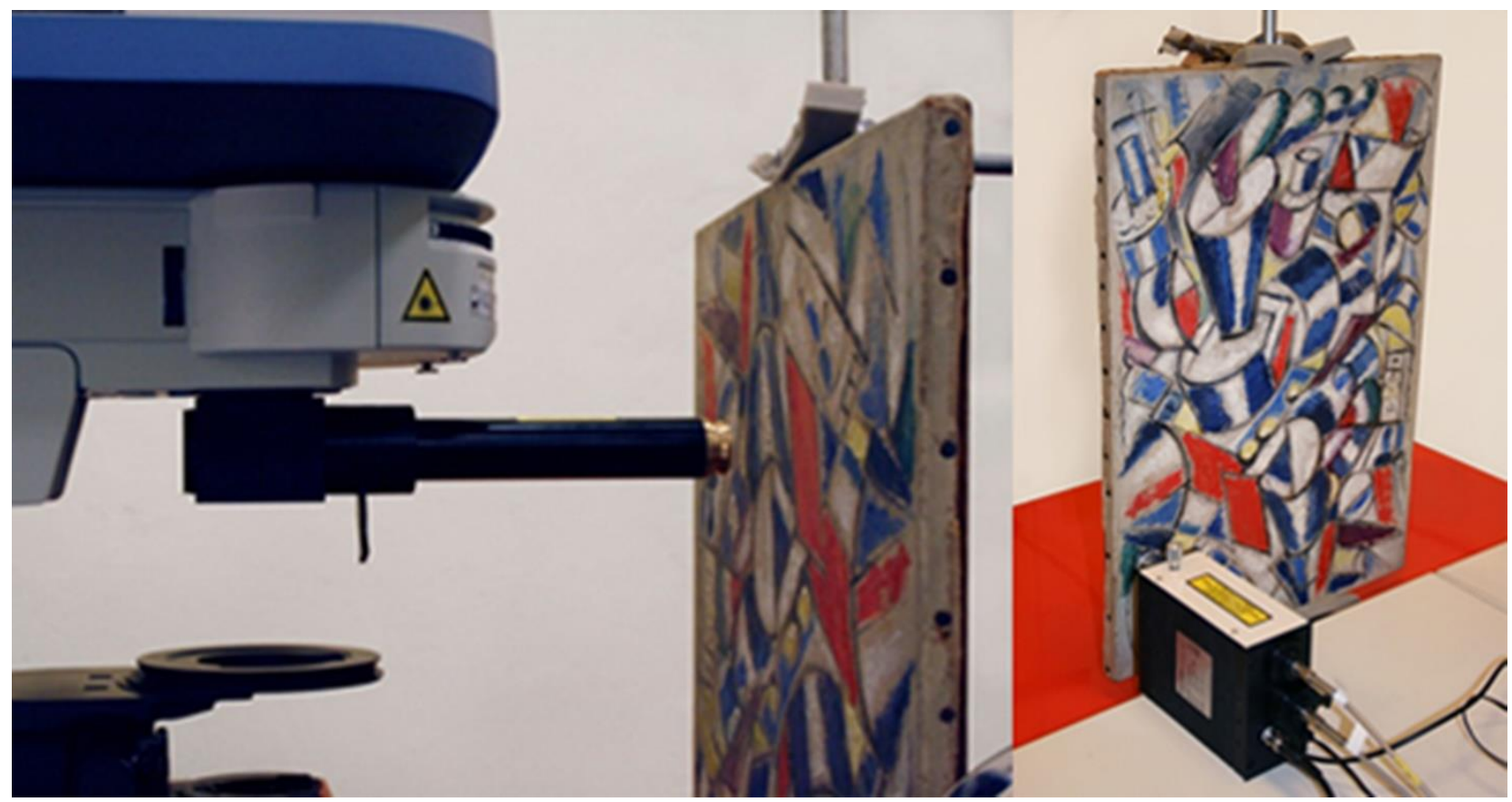

Fig 3. Device including $45^{\circ}$ inclined mirror and tube and fibre optic configuration

Table 2. ESEM chemical mapping of the six pure pigments pellets. The shown chemical elements are indicated in the respective colour for each map.

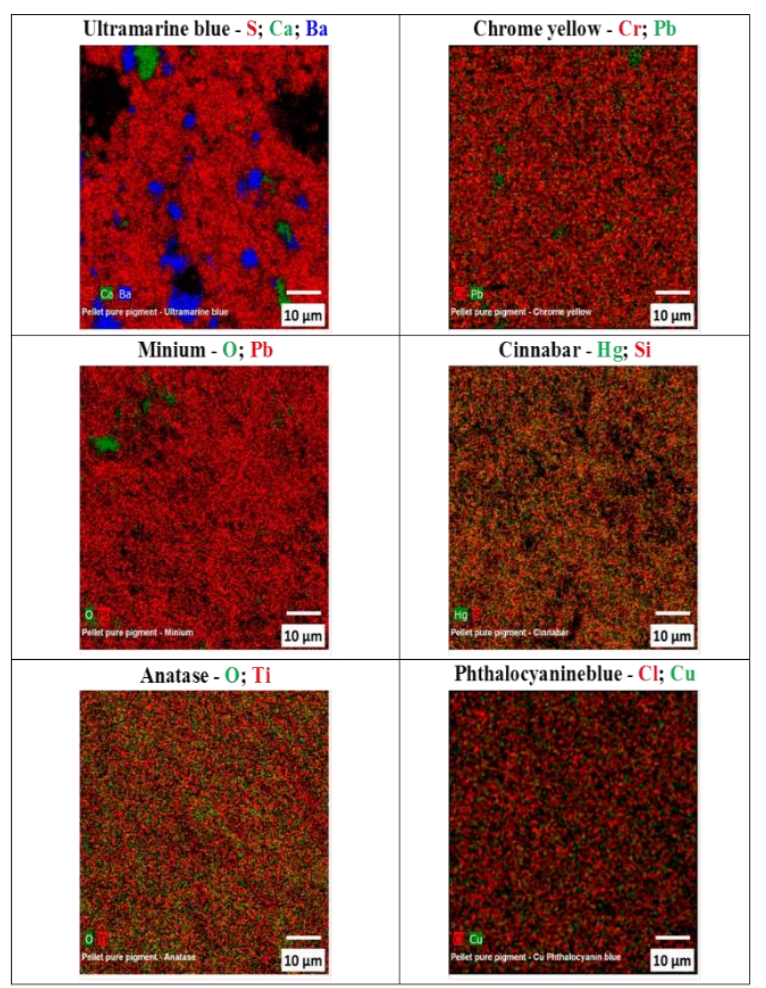

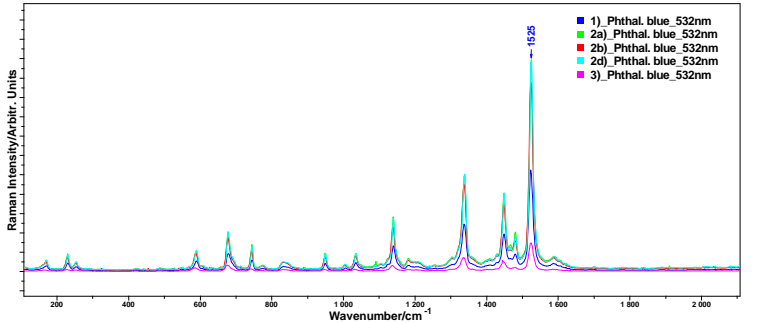

Fig 4. Uncorrected Raman spectra of Phthalocyanine with setups $1,2 \mathrm{a}, 2 \mathrm{~b}, 2 \mathrm{~d}, 3$

In the following graphics (Fig 5 and Fig 6), the intensities of each set-up and for each pigment are compared to those obtained with the setup 1 (traditional microscope, configuration 1 in Fig 2) normalized to 1.

The performance index for anatase on a pure pigment was calculated as a LODC, calculated as described in the equations (eq.1-6). The results are summarised in detection of a set-up but are used as index to compare the performance of the set-ups. For a more realistic estimation, other experiments are needed and a more precise determination of $b$ is needed in table 3 and are based on the average of three measurements. The LODC values are not believed to give a realistic limit of the values for the LODC of the configurations 1 and 2 values are lower than for configuration 3 . These values confirm the trend of the performance observed during the experiments with various set-ups 


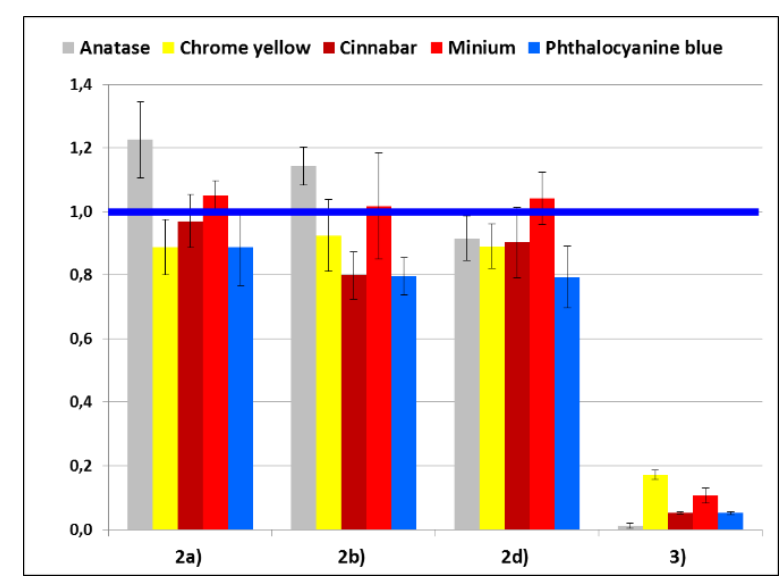

Fig 5. Intensities (average of 3) normalised to the performance of configuration 1 measured with each set-up with the $532 \mathrm{~nm}$ Laser.

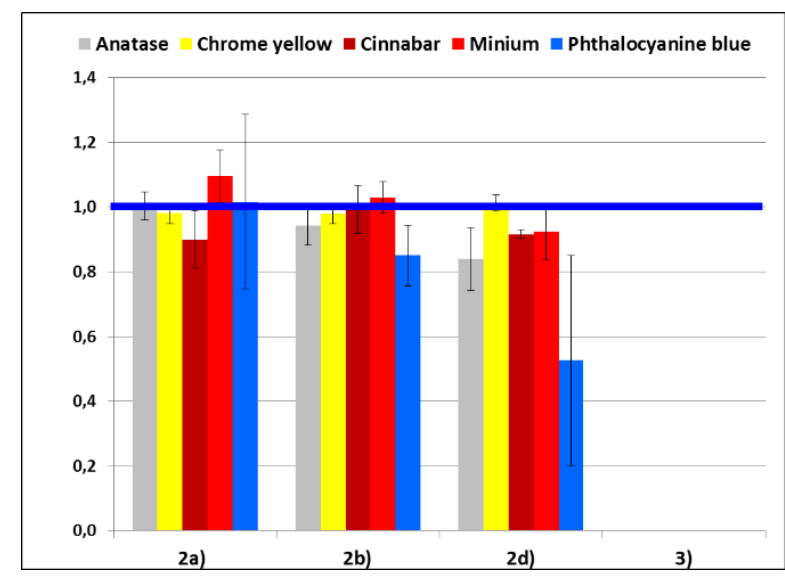

Fig 6. Intensities normalised to the performance of configuration 1 from each set-up with the $785 \mathrm{~nm}$ Laser.

Table 3. $\mathrm{LOD}_{\mathrm{I}}, \mathrm{LOD}_{\mathrm{C}}$ and $\mathrm{b}$ for pure pigment calculated for anatase measured with lasers $532 \mathrm{~nm}$ and $785 \mathrm{~nm}$ (all measurements given for $10 \%$ transmission filter)

\begin{tabular}{|c|c|c|c|c|c|c|}
\hline \multirow{2}{*}{ Set-up } & \multicolumn{3}{|c|}{ Laser $532 \mathrm{~nm}$} & \multicolumn{3}{|c|}{ Laser $785 \mathrm{~nm}$} \\
\hline & $\begin{array}{l}\text { LODI } \\
\text { (Cnts/s) }\end{array}$ & $\begin{array}{l}\text { LODC } \\
\text { (wt } \%)\end{array}$ & $\begin{array}{c}\mathrm{b} \text { pure anatase } \\
(\mathrm{Cnts} / \mathrm{s} . \mathrm{wt} \%)\end{array}$ & $\begin{array}{l}\text { LODI } \\
\text { (Cnts/s) }\end{array}$ & $\begin{array}{l}\text { LODC } \\
\text { (wt } \%)\end{array}$ & $\begin{array}{c}\text { b pure anatase } \\
\text { (Cnts/s.wt } \%)\end{array}$ \\
\hline 1 & $274 *$ & $0.1 *$ & $2690 *$ & 69 & 0.4 & 165 \\
\hline $2 \mathrm{a}$ & 304 & 0.1 & 3113 & 67 & 0.4 & 171 \\
\hline $2 b$ & 301 & 0.1 & 2928 & 70 & 0.4 & 159 \\
\hline $2 \mathrm{c}$ & \multicolumn{6}{|c|}{ Setup not available for these measurements } \\
\hline $2 d$ & 295 & 0.1 & 2488 & 70 & 0.5 & 142 \\
\hline 3 & 22 & 8.0 & 3 & Setup not av & these $n$ & nents \\
\hline
\end{tabular}

$* 1 \%$ filter was used; intensity values were multiplied by ten

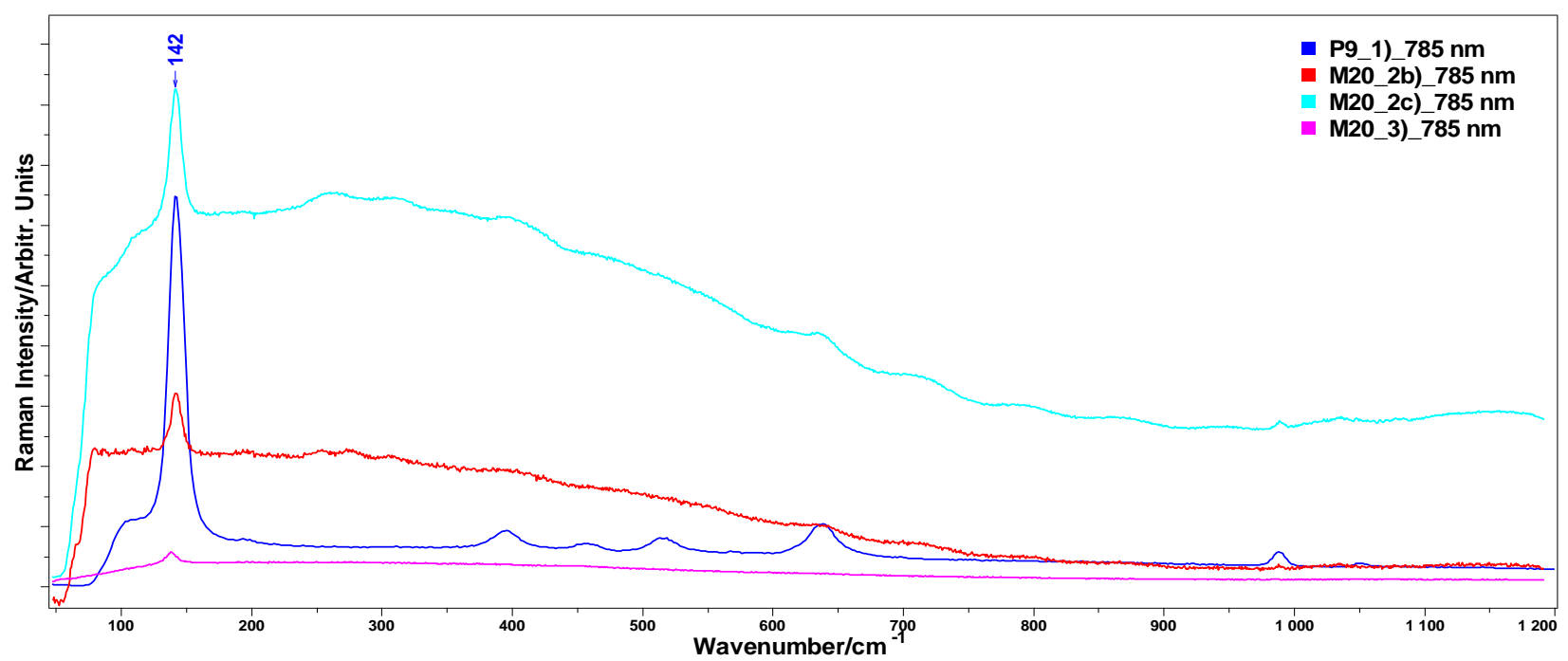

Fig 7. Uncorrected Raman spectra of sample P9 (setup 1) and spot M20 (setups 2b, 2c, 3) 


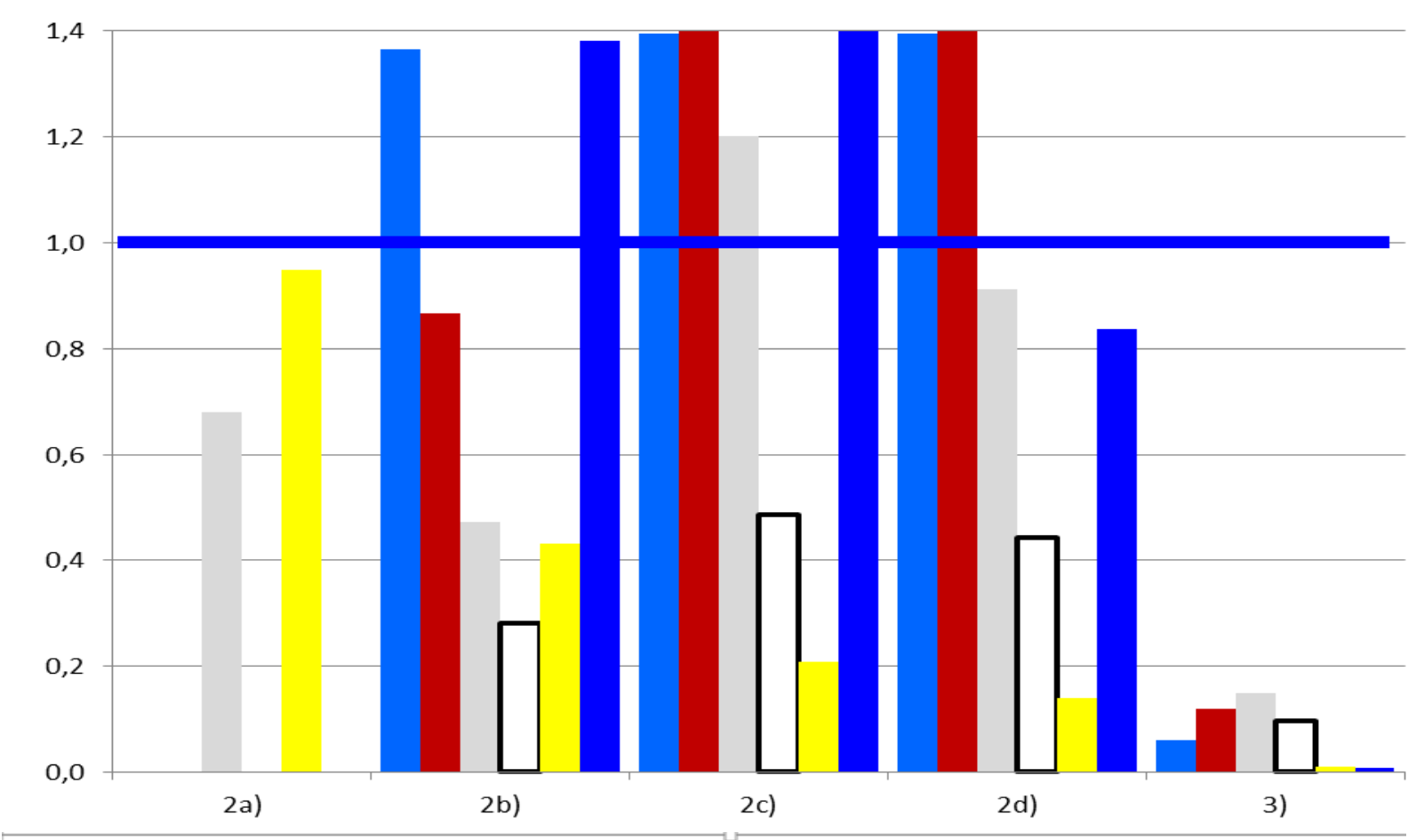

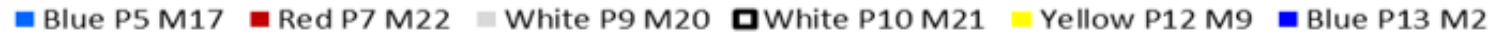

Fig 8. Intensity normalized to performance of configuration 1 from each setup

\subsection{Case study}

The pigments identified were: anatase, lazurite/ultramarine, mangan violet, cinnabar, minium, baryte, chrome yellow and $\mathrm{Cu}$ phthalocyanine blue. Example spectra of anatase obtained with different setups are seen in Fig 7.

The intensities from the most intense pigment lines obtained from the 'Cubist still life' are shown in the following (Fig 8). The values show much more variations than those for the reference samples. Intensities from some for non-invasive configurations are even higher than those obtained with the traditional microscope setup on a collected sample.
As for the pure pigment the LOD for anatase was calculated and is shown in table 4. A value for the configurations $2 \mathrm{c}$ and 3 are not available as the corresponding $b$ values were not available. The $\operatorname{LOD}_{\mathrm{C}}$ values for configurations $1,2 \mathrm{a}, 2 \mathrm{~b}$ and $2 \mathrm{~d}$ were all slightly higher than compared to the results on the pure anatase. With values from $0.3 \mathrm{wt} \%$ to $0.8 \mathrm{wt} \%$ the $\mathrm{LOD}_{\mathrm{C}}$ value indicate still a better performance than the external measuring head on the pure pigment with $8 \mathrm{wt} \%$.

Table 4. $\mathrm{LOD}_{\mathrm{I}}$ taken from the background of the measurements of the case study, $\mathrm{LOD}_{\mathrm{C}}$ and $\mathrm{b}$ for pure pigment calculated for anatase measured with the laser $785 \mathrm{~nm}$

\begin{tabular}{|c|c|c|c|}
\hline \multirow{2}{*}{ Set-up } & \multicolumn{3}{|c|}{ Laser $785 \mathrm{~nm}$} \\
\hline & $\mathrm{LOD}_{\mathrm{I}}(\mathrm{Cnts} / \mathrm{s})$ & $\mathrm{LOD}_{\mathrm{C}}(\mathrm{wt} \%)$ & $\mathrm{b}_{\text {pure anatase }}(\mathrm{Cnts} / \mathrm{s}$.wt $\%)$ \\
\hline 1) & 47 & 0.3 & 165 \\
\hline 2a) & 91 & 0.5 & 171 \\
\hline 2b) & 72 & 0.4 & 159 \\
\hline 2c) & & vailable for this & \\
\hline 2d) & 117 & 0.8 & 142 \\
\hline 3) & & vailable for this & \\
\hline
\end{tabular}




\subsection{Comparison of the set-ups}

The use of the procedure established in this article to calculate the $\mathrm{LOD}_{\mathrm{C}}$ value for the comparison of the performance of an experimental set-up gives us the possibility to benchmark the various experimental configurations with regard to the obtained signal and background intensities.

All instrumental configurations allowed identifying the pigments anatase, chrome yellow, minium, cinnabar and copper-phthalocyanine blue in the samples. Depending on the set-up, variations were found in the intensity of the signals of the pigments. The horizontal beam configurations ( $2 \mathrm{a}, 2 \mathrm{~b}$ and $2 \mathrm{~d})$ on the reference samples showed spectral responses of 0.8 to 1.2 of the intensities measured with the microscope (configuration 1). The intensities from the fibre optics (configuration 3) dropped to 0.1 . The intensities from the horizontal beam set-ups were comparable to the microscope whether the use of fibre optics introduced a significant loss of signal.

This drop of intensity leads also to a less favourable $\mathrm{LOD}_{\mathrm{C}}$ value for the configuration 3. All configurations without external measuring head gave a LOD $_{C}$ value of about $0.1 \mathrm{wt} \%$ in contrast to the value of $8 \mathrm{wt} \%$ for the configuration 3 . These results show the potential of horizontal beam set-ups for the analyses of artworks as their performance is equivalent to the traditional microscope configuration. On the painting, the noninvasive response from the horizontal beam set-ups (2a-d) shows much larger fluctuations in intensity with respect to the microscope configurations: from 0.15 to 1.85 . Compared to the reference samples, the paint layer of the painting is much more heterogeneous as it is composed of pigment mixed with filler and binding media at $\mu \mathrm{m}$ scale. This induces a momentum of random to the composition of the analysed volume, influencing the intensity regardless of the set-up used. Again, the intensities of the fibre optic set-up dropped to ca. 0.1. This suggests that the choice of the measurement spot on the painting is more important than the choice between the different set-ups of configuration 1 and 2. However, comparing configurations 1 and 2 , difficulties in positioning the horizontal beam set-up can hamper the acquisition of a good spectrum. Either the Raman spectrometer or the artwork has to be moved in a $\mu \mathrm{m}$ scale to position the laser spot to the area of interest. This requires often improvements of the experiment, especially regarding the mechanical stability of the analytical set-up as well as the artwork and its support or the use of a precision motor driven stage to move the art work.

\section{Conclusions}

In this work, different non-invasive micro-Raman setups were compared to the traditional microscope set-up with respect to their spectral response efficiency. For the non-invasive measurements, four horizontal beam set-ups and an external measuring head connected to the spectrometer by fibre optics were used. Using limit of detection values as index, their performances were compared. Reference pigment samples and a painting were studied.

The horizontal beam configurations showed spectral responses in signal intensity very similar to those of the traditional microscope. These results show the potential of horizontal set-ups for the analyses of artworks, as their performance is equivalent to the traditional microscope configuration. Neither the laser's path deviation nor the extension tubes (self-made or commercial) affected significantly the spectral response.

All measurements performed with the fibre optics' setup originated a significant lower spectral response (lower signal intensity in the range of $10 \%$ compared to the other configurations). These are the finds of our equipment and may not be generalized to all fibre based configurations. .

The mode of operation for measurements on the painting was quite different from the measurement on paint samples. Working with the traditional set-up allows a much better choice of the measurement spot. It is possible to avoid binding media, dirt and other extraneous materials or layers and to focus on a spot, which contains a high proportion of pigment. In contrast, non-invasive measurements with the horizontal set-ups and the fibre optics measurement head are bound to access the painting via its surface with all its dirt and varnish layers. Therefore, it was more difficult to focus on a pigment grain.

The movement of the object relative to the focal spot of the Raman spectrometer requires a precision in positioning in the range of several micrometres, which is difficult to obtain without the help of a precision motordriven xyz-stage. An additional challenge working with a canvas painting is to keep the object steady, especially the canvas in the central area of the painting can vibrate, during the measurements, which is very difficult to prevent under the necessary precautions to prevent mechanical damage to the painting.

Despite the challenge of positioning set-up in front of the object, any of the horizontal beam set-ups are proficient options for a non-invasive measurement. An inherent advantage of the non-invasive approach is the possibility to probe much more spots on the objects as in would be possible with a sampling. Therefore, the 
horizontal beam set-up allows to obtain a much more complete understanding of the pigments palette used without the risk that samples to not represent the full pigments palette and at the same time using a very effective configuration of the Raman spectrometer with high spectral response.

\section{Acknowledgements}

We would like to acknowledge Christoph Herm (HfBK Dresden), Jens Bartoll (SPSG Potsdam), René

\section{References}

Aceto, M., Agostino, A., Fenoglio, G., Baraldi, P., Zannini, P., Hofmann, C., \& Gamillscheg, E. (2012). First analytical evidences of precious colourants on Mediterranean illuminated manuscripts. Spectrochimica Acta. Part A: Molecular and Biomolecular Spectroscopy, 95, 235-245.

Bell, I. M., Clark, R. J., \& Gibbs, P. J. (1997). Raman spectroscopic library of natural and synthetic pigments (pre- approximately 1850 AD). Spectrochimica Acta. Part A: Molecular and Biomolecular Spectroscopy, 53A(12), 2159-2179.

Bersani, D., Lottici, P. P., Vignali, F., \& Zanichelli, G. (2006). A study of medieval illuminated manuscripts by means of portable Raman equipments. Journal of Raman Spectroscopy : JRS, 37(10), 1012-1018.

Boschetti, C., Corradi, A., \& Baraldi, P. (2008). Raman characterization of painted mortar in Republican Roman mosaics. Journal of Raman Spectroscopy: JRS, 39(8), 1085-1090.

Bouchard, M., \& Smith, D. C. (2003). Catalogue of 45 reference Raman spectra of minerals concerning research in art history or archaeology, especially on corroded metals and coloured glass. Spectrochimica Acta. Part A: Molecular and Biomolecular Spectroscopy, 59(10), 2247-2266.

Burgio, L., \& Clark, R. J. H. (2001). Library of FT-Raman spectra of pigments, minerals, pigment media and varnishes, and supplement to existing library of Raman spectra of pigments with visible excitation. Spectrochimica Acta. Part A: Molecular and Biomolecular Spectroscopy, 57(7), 1491-1521.

Clark, R. J. H. (2002). Pigment identification by spectroscopic means: An arts/science interface. Comptes Rendus. Chimie, 5(1), 7-20.

Deneckere, A., Schudel, W., Van Bos, M., Wouters, H., \& Bergmans, A. (2010). A., P. Vandenabeele, L. Moens. Spectrochimica Acta. Part A: Molecular and Biomolecular Spectroscopy, 75, 511-519.
Allonge (LKA Berlin) and Dieter Breidt (SPK, Berlin) for their support of this work.

\section{Conflict of Interest}

Authors declared no conflict of interest.

Pérez-Alonso, M., Castro, K., Martinez-Arkarazo, I., Angulo, M., Olazabal, M. A., \& Madariaga, J. M. (2004). Analysis of bulk and inorganic degradation products of stones, mortars and wall paintings by portable Raman microprobe spectroscopy. Analytical and Bioanalytical Chemistry, 379(1), 4250 .

Reiche, I., Pages-Camagna, S., \& Lambacher, L. (2004). In situ Raman spectroscopic investigations of the adorning gemstones on the reliquaryHeinrich's Cross from the treasury of Basel Cathedral. Journal of Raman Spectroscopy: JRS, 35(8-9), 719-725.

Röhrs, S., \& Stehr, U. (2014). Berliner Beiträge zur Archäometrie. Kunsttechnologie und Konservierungswissenschaft, 24, 47-55.

Scherrer, N. C., Zumbuehl, S., Delavy, F., Fritsch, A., \& Kuehnen, R. (2009). Synthetic organic pigments of the 20th and 21st century relevant to artist's paints: Raman spectra reference collection. Spectrochimica Acta. Part A: Molecular and Biomolecular Spectroscopy, 73(3), 505-524.

Smith, G. D., \& Clark, R. J. H. (2004). Raman microscopy in archaeological science. Journal of Archaeological Science, 31(8), 1137-1160.

Trentelman, K., \& Turner, N. (2009). Investigation of the painting materials and techniques of the late-15th century manuscript illuminator Jean Bourdichon. Journal of Raman Spectroscopy: JRS, 40(5), 577584.

Vandenabeele, P. (2013). Practical Raman Spectroscopy: An Introduction. Wiley.

Vandenabeele, P., \& Moens, L. (2012). Some ideas on the definition of Raman spectroscopic detection limits for the analysis of art and archaeological objects. Journal of Raman Spectroscopy: JRS, 43(11), 15451550. 
Vandenabeele, P., Castro, K., Hargreaves, M., Moens, L., Madariaga, J. M., \& Edwards, H. G. M. (2007). Comparative study of mobile Raman instrumentation for art analysis. Analytica Chimica Acta, 588(1), 108-116.

Vandenabeele, P., Edwards, H. G. M., \& Moens, L. (2007). A decade of Raman spectroscopy in art and archaeology. Chemical Reviews, 107(3), 675-686.
Vandenabeele, P., Lambert, K., Matthys, S., Schudel, W., Bergmans, A., \& Moens, L. (2005). In situ analysis of mediaeval wall paintings: A challenge for mobile Raman spectroscopy. Analytical and Bioanalytical Chemistry, 383(4), 707-712.

Ziemann, M. A. (2006). In situ micro-Raman spectroscopy on minerals on-site in the Grotto Hall of the New Palace, Park Sanssouci, in Potsdam. Journal of Raman Spectroscopy: JRS, 37(10), 10191025.

\section{How to Cite This Article:}

Aibéo C, Röhrs S, Marucci G, Reiche I. Comparison of Different Non-invasive $\mu$-Raman Set-ups for Studying Pigments in Art Works. Ir Cons Sci J. 2017; 1 (1) :6-16

URL: http://journal.richt.ir/ics/article-1-22-en.html 